\title{
Thermodynamic Study of Fatty Soil Removal from Polyester Fiber by Liquid Chromatography with Fabric Column
}

\author{
Hisako TAgaya ${ }^{1 *}$, Satomi Tsuda ${ }^{1 \dagger}$ and Ken HigashitsujI ${ }^{2}$ \\ ${ }^{1}$ Faculty of Education, Shiga University \\ (2-5-1 Hiratsu, Otsu 520-0862, JAPAN) \\ ${ }^{\dagger}$ Present Address: 18-27 Hirosawaikeshitacho, Saga, Ukyo-ku, Kyoto 616-8307, JAPAN \\ 2 (232 Buejincho, Sawaragicho-agaru, Muromachi-doori,Kamigyou-ku, Kyoto 602-8029, JAPAN)
}

Edited by M. Iwahashi, Kitasato Univ., and accepted December 2, 2003 (received for review September 8, 2003)

\begin{abstract}
The mechanisms for removal of synthetic sebum constituents squalene, cholesterol, triolein and oleic acid from polyester (PET) fabric substrate in an aqueous solution of sodium dodecyl sulfate (SDS) were studied by applying a pseudo-phase retention equation to the migration of a soil in a liquid chromatography column in which the fabric serves as the stationary phase and an aqueous micellar solution of SDS serves as the mobile phase. The retention volume of the four fatty soils on PET fabric was measured as a function of the SDS concentration in the mobile phase. The changes in standard free energy of soil transfer from water onto the fabric substrate $\left(-\Delta G_{\mathrm{sw}}^{\circ}\right)$, from water into micelles $\left(-\Delta G_{\mathrm{mw}}^{\circ}\right)$ and from fabric substrate into the micelles $\left(-\Delta G_{\mathrm{ms}}^{\circ}\right)$ were calculated from the partition coefficient data determined chromatographically at various concentrations of micellar mobile phases. The $-\Delta G^{\circ}{ }_{\text {sw }}$ for the re-deposition of fatty soil onto the PET substrate increased in the following order: cholesterol $<$ oleic acid $<$ squalene $<$ triolein. The $-\Delta G^{\circ}{ }_{\mathrm{mw}}$ for solubilization of the soil from water (non-micellar solution) into SDS micelles increased in the following order: oleic acid $<$ cholesterol $<$ squalene $<$ triolein. The $-\Delta G^{\circ}$ ms for removal of the soil from the PET substrate into SDS micelles decreased in the following order: cholesterol $>$ squalene $>$ triolein. These thermodynamic parameters suggest that removal of fatty soils from PET fabric is dependent on the tendencies of the soils to redeposit onto the fabric and to solubilize into SDS micelles.
\end{abstract}

Key words: fatty soil removal, liquid chromatography, fabric column, aqueous micellar mobile phase, standard free energy change

\section{Introduction}

Contact of fabric surfaces with human skin inevitably results in transfer of human sebum, which cannot be easily removed by washing the fabrics in water with conventional detergents (1). Sebum, the sebaceous secretion of the skin, consists of a complex mixture of triglycerides, squalene, cholesterol and other sterols, free fatty acids and aliphatic hydrocarbons (2) and is the major oily soil constituent in home laundry.
Although many studies on the removal of sebum soil from different substrates have been reported, few address the thermodynamics of sebum soil removal from fiber substrates into aqueous surfactant solutions. The mechanism for removal of oily soil by a substratewater-micelle system can be considered a two-step process involving the transfer of soil from substrate to water (non-micellar phase) and then from water into micelles. Thus, the free energy change of soil determines whether a detergent process will be successful in removing the

\footnotetext{
${ }^{*}$ Correspondence to: Hisako TAGAYA, Faculty of Education, Shiga University, 2-5-1 Hiratsu, Otsu 520-0862, JAPAN

E-mail: tagaya@sue.shiga-u.ac.jp
} 
soil from substrate.

Previously, we proposed a thermodynamic approach for estimating soil removal from substrates, that is, the transfer of an oil-soluble dyestuff as the oily soil model from a substrate into an aqueous surfactant solution, using a chromatographic method whereby the yarn filament or the pulverized form of a substrate serves as the stationary phase and a detergent solution serves as the mobile phase $(3,4)$. This method has made it possible to characterize the soil removal mechanism thermodynamically without mechanical force. There, however, have been significant differences in surface properties between the yarn filament form and the powdered form of a substrate. Sebum soil found on clothes has been very impossible to remove from polyester (PET) fabric especially using an aqueous laundry system (1). To understand removal of sebum soil from PET fabric surfaces, it is necessary to investigate more precisely the complex interactions between the sebum soil and the PET fabric or aqueous surfactant solution.

Here, we describe the mechanism of transfer of synthetic sebum soil constituents from PET fabric substrate into an aqueous solution of sodium dodecyl sulfate (SDS) determined by a liquid chromatography method with PET fabric used as the stationary phase. We calculated the partition coefficients and the standard free energy changes for transfer of synthetic sebum constituents between polyester fabric and SDS micelles and discuss the relative ease with which the constituents are removed and re-deposited on the fabric.

\section{Experimental}

\subsection{Materials}

The stationary phase comprised of plain-woven white fabric (without optical brightener) made from 100\% PET filament yarn (JIS L-0803, Japanese Standards Association) (Table 1). SDS anionic surfactant standard reagent (99.2\% purity), was obtained from Nacalai Tesque, Inc., Kyoto, Japan, and was used without further purification. Guaranteed grade squalene (98\% purity), cholesterol (99\% purity), triolein (99\% purity) and oleic acid (99\% purity) obtained from Nacalai Tesque, Inc., were used as the model sebum soil constituents without further purification (Scheme 1). Extra puregrade CI Solvent Yellow 5 (SY5, 1-phenylazo-2-naphthylamine) used for the model colored oily soil was obtained from Nacalai Tesque, Inc. and was purified as described previously (4) (Scheme 1). HPLC-grade water from Nacalai Tesque, Inc., was used as the bulk solvent for the micellar mobile phase. All other reagents were of guaranteed grade and used without further purification.

\section{$\mathbf{2} \cdot 2$ Methods}

The apparatus and procedure used for liquid chromatography (LC) were fundamentally the same as those reported previously (4). A degasser (model ILD, Waters Co., Milford, MA, USA) for removing dissolved gases from mobile phase was connected between the solution reservoir and the inlet to the pump to decrease baseline drift or shifts and background absorbance at low wavelengths, and to improve detection sensitivity. A 5-mm i.d. $\times$ 50-mm-long glass column (model 021901, Waters Co.) was used for chromatography. The column was packed with 560 sheets of PET fabric disks of the same diameter in the dry state as the interior of the column. The packing in the columns was purified as described previously (4). The flow rate of the PET column was 2 $\mathrm{mL} / \mathrm{min}$. The detector wavelengths were $277 \mathrm{~nm}$ for squalene, $285 \mathrm{~nm}$ for cholesterol, $280 \mathrm{~nm}$ for oleic acid, $230 \mathrm{~nm}$ for triolein and $445 \mathrm{~nm}$ for SY5. Elution volumes for the column were determined for a range of SDS concentrations. Each model soil was introduced individually onto the top of the column as an ethanol solutions containing $0.1 \%$ squalene, cholesterol, or triolein $(\mathrm{w} / \mathrm{v})$ or $2 \%$ oleic acid $(\mathrm{w} / \mathrm{v})$, or as a solution of ethanol and water $(50 / 50 \mathrm{v} / \mathrm{v})$ containing $0.01 \%$ SY5 $(\mathrm{w} / \mathrm{v})$. These sample solutions were applied by syringe to the column with an injector fitted with a $5-\mu \mathrm{L}$ sample loop (model 7755-065, Waters Co.).

The volume of the stationary phase $\left(V_{\mathrm{s}}\right)$ was calculated as the difference between the total column volume $\left(V_{\mathrm{t}}\right)$ and the void volume $\left(V_{\mathrm{m}}\right)$ of the column (4). The

Table 1 Characterization of Plain Weave Fabric Used as the Stationary Phase.

\begin{tabular}{|c|c|c|c|c|c|c|}
\hline Fabric & Description & $\begin{array}{c}\text { Filament } \\
\text { warp }\end{array}$ & $\begin{array}{l}\text { Yarn Denier } \\
\text { weft }\end{array}$ & $\begin{array}{l}\text { Threads } / \mathrm{cm} \\
\text { warp } \times \text { weft }\end{array}$ & $\begin{array}{l}\text { Mass } \\
\left(\mathrm{g} / \mathrm{m}^{2}\right)\end{array}$ & $\begin{array}{c}\text { Thickness } \\
\text { (mm) }\end{array}$ \\
\hline Polyester & $100 \%$ Polyester fiber & 75 & 75 & $42 \times 38$ & 70 & 0.0786 \\
\hline
\end{tabular}


void proportion $\left(V_{\mathrm{m}} / V_{\mathrm{t}}\right)$ of the columns packed with PET fabric was $61 \%$ or $72 \%$. $V_{\mathrm{m}}$ was always measured before and after each experiment and did not change during any experiment. The streaming potential measurements were the same as those reported previously (3). The $\mathrm{pH}$ of the aqueous SDS solutions was usually 5.5-5.6 as determined with a $\mathrm{pH}$ meter. All experiments were conducted in a constant temperature room at $20^{\circ} \mathrm{C}$.
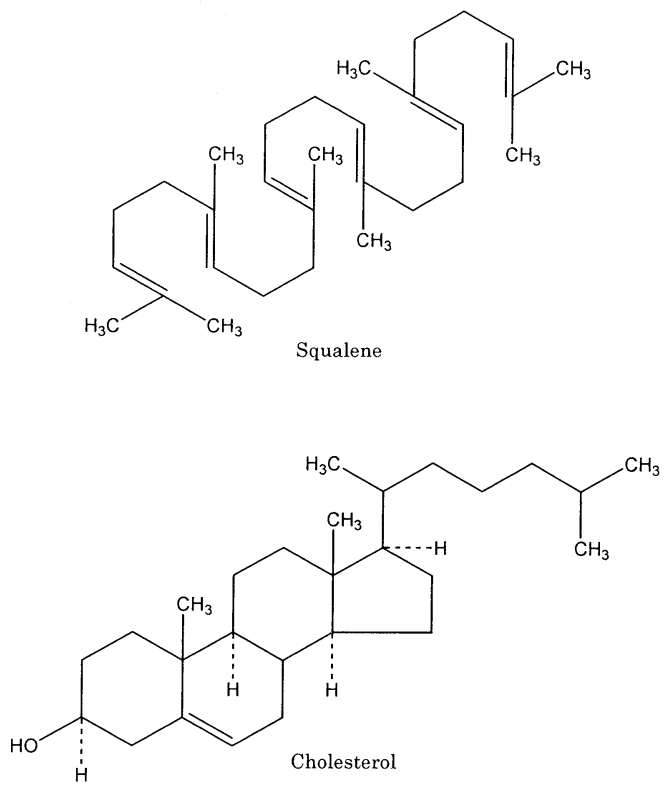

$\mathrm{CH}_{3}\left(\mathrm{CH}_{2}\right)_{7} \mathrm{CH}=\mathrm{CH}\left(\mathrm{CH}_{2}\right)_{7} \mathrm{C} \rightleftharpoons \mathrm{OH}$

Oleic Acid

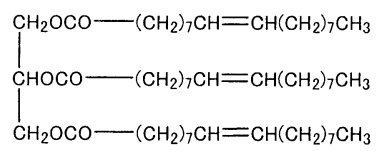

Triolein

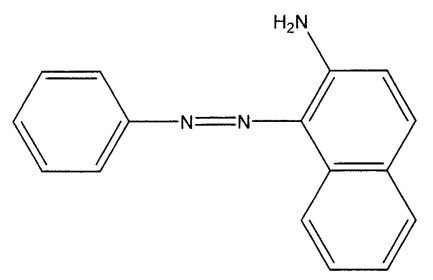

Solvent Yellow 5 (SY5, 1-phenylazo-2-naphthylamine)

Scheme 1 Chemical Structures of Model Soils.

\section{Results}

Chromatograms of each fatty soil and the oil-soluble dyestuff SY5 eluted from the PET fabric column with each SDS solution are shown in Figs. 1-5. An initial peak corresponded to the sample solvent (ethanol) of the SY5 or fatty soil. The elution curves of SY5 form PET fabric column (Fig. 1) were smooth and did not have a ripple waveform such as that produced with the filament yarn column (3). Because absorbance of fatty soils in the ultraviolet region is very weak, the elution peaks for the fatty soils were much smaller than those for SY5. All fatty soils and SY5 were eluted at SDS concentrations above the critical micellar concentration (cmc) of $8.2 \times 10^{-3} \mathrm{~mol} \mathrm{dm}^{-3}$ (5). The elution volume $\left(V_{\mathrm{e}}\right)$ for the fatty soils and SY5 decreased with increase in the SDS concentration.

The corresponding capacity factors $\left(k^{\prime}=\left(V_{\mathrm{e}}-V_{\mathrm{m}}\right)\right.$

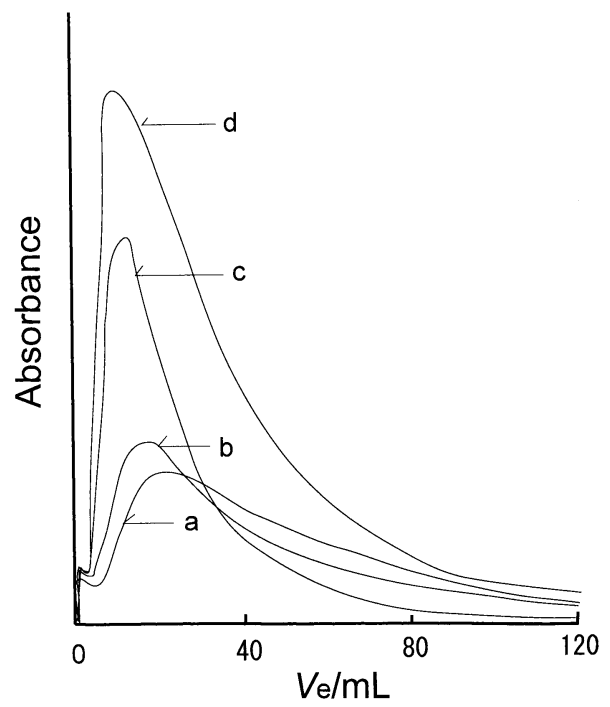

Fig. 1 Effect of SDS Concentrations in the Mobile Phase on Chromatogram of Solvent Yellow 5 Eluted from Polyester Fabric Column.

SDS Concentration: (a); $8.5 \times 10^{-3} \mathrm{~mol}$ $\mathrm{dm}^{-3},(\mathrm{~b}) ; 8.75 \times 10^{-3} \mathrm{~mol} \mathrm{dm}^{-3}$, (c); 9.0 $\times 10^{-3} \mathrm{~mol} \mathrm{dm}^{-3},(\mathrm{~d}) ; 9.25 \times 10^{-3} \mathrm{~mol}$ $\mathrm{dm}^{-3}$

Flow Rate: $2.0 \mathrm{~mL} \mathrm{~min}{ }^{-1}$, Wavelength: $445 \mathrm{~nm}$, Sample Volume: $5 \mu \mathrm{L}$ of Solution in Ethanol and Water $(50 / 50$ v/v) Containing $0.01 \%$ Solvent Yellow 5 $(\mathrm{w} / \mathrm{v})$. 


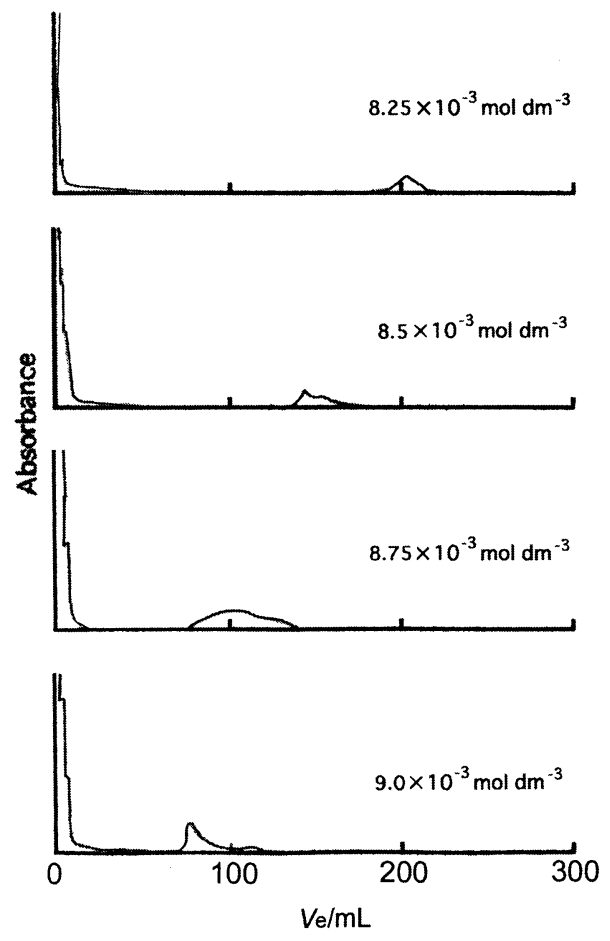

Fig. 2 Effect of SDS Concentrations in the Mobile Phase on Chromatogram of Cholesterol Eluted from Polyester Fabric Column.

Flow Rate: $2.0 \mathrm{~mL} \mathrm{~min}^{-1}$, Wavelength: $285 \mathrm{~nm}$, Sample Volume: $5 \mu \mathrm{L}$ of Ethanol Solution Containing 0.1\% Cholesterol (w/v).

$/ V_{\mathrm{m}}$ ) for PET fabric columns are shown in Table 2. The $k^{\prime}$ for squalene, triolein, oleic acid, cholesterol and SY5 on PET fabric substrate decreased with increasing SDS concentrations above the cmc. This is similar to that of oil-soluble dyestuffs on yarn filament or fiber powder substrates, which have been used as oily soil models $(3,4)$. It is clear that the $k^{\prime}$ values are affected by the solubilization of the fatty soils or SY5 into SDS micelles. The $k^{\prime}$ values for the fatty soils and SY5 at the same concentrations of SDS increased in the following order: SY $5 \ll$ cholesterol $<$ squalene $<$ oleic acid $\ll$ triolein. These results suggest that the $k^{\prime}$ value depends strongly on the ease of removal of each fatty soil or SY5 from the PET fabric substrate into the aqueous SDS micelle solution. The $k^{\prime}$ value for SY5 on PET fabric was slightly less than the $k^{\prime}$ value obtained on PET yarn filament (3), suggesting properties of the PET surfaces differ slightly between the fabric used in the present work and yarn filament used in the previous report. From

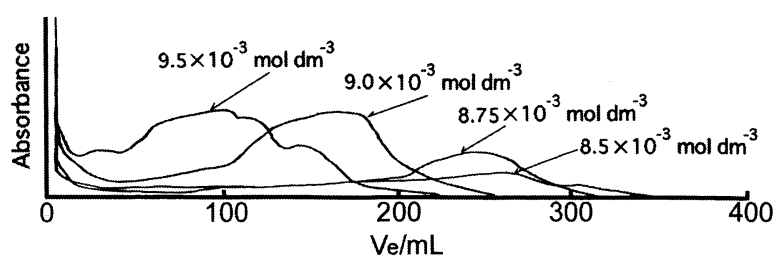

Fig. 3 Effect of SDS Concentrations in the Mobile Phase on Chromatogram of Squalene Eluted from Polyester Fabric Column.

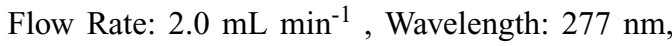
Sample Volume: $5 \mu \mathrm{L}$ of Ethanol Solution Containing $0.1 \%$ Squalene (w/v).

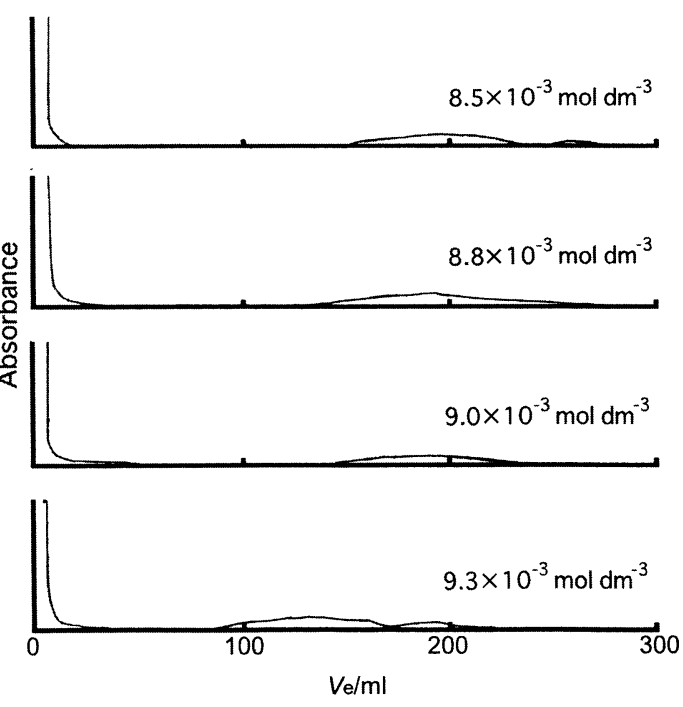

Fig. 4 Effect of SDS Concentrations in the Mobile Phase on Chromatogram of Oleic Acid Eluted from Polyester Fabric Column.

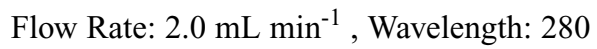
$\mathrm{nm}$, Sample Volume: $5 \mu \mathrm{L}$ of Ethanol Solution Containing 2.0\% Oleic Acid $(\mathrm{w} / \mathrm{v})$.

these results, it is inferred that the intensity of interaction between the fatty soils and SY5 and PET fabric substrate or aqueous SDS micelle solution alters the behavior of elution from the PET fabric column.

\section{Discussion}

\section{4-1 Thermodynamics of the Fatty Soil Removal Process}

In liquid chromatography, the elution behavior of a solute eluted with aqueous micellar mobile phase is dependent on the combined effects of three partition 


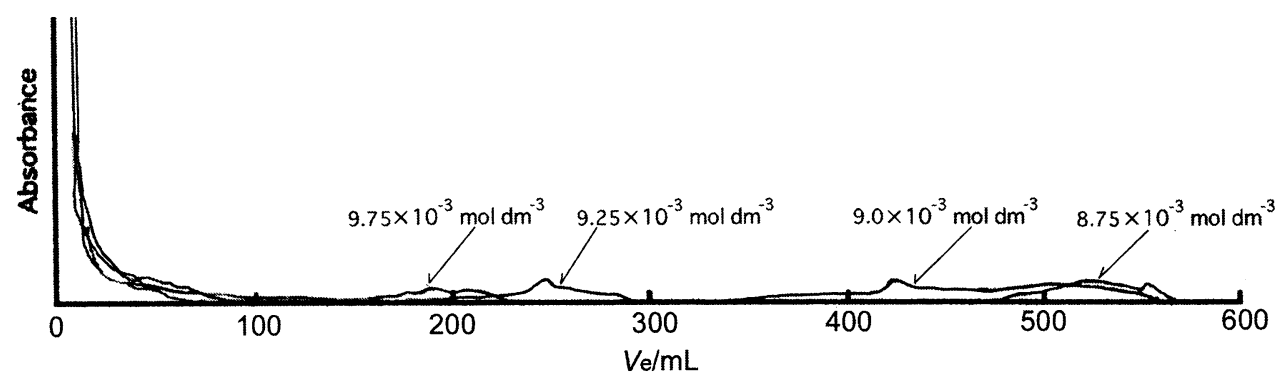

Fig. 5 Effect of SDS Concentrations in the Mobile Phase on Chromatogram of Triolein Eluted from Polyester Fabric Column.

Flow Rate: $2.0 \mathrm{~mL} \mathrm{~min}{ }^{-1}$, Wavelength: $285 \mathrm{~nm}$, Sample Volume: $5 \mu \mathrm{L}$ of Ethanol Solution Containing $0.1 \%$ Triolein (w/v).

Table 2 Variation of Capacity Factors of Fatty Soils from a Polyester Column as a Function of SDS Concentration in the Mobile Phase.

\begin{tabular}{|c|c|c|c|c|c|c|c|c|c|c|c|c|c|}
\hline \multirow[b]{2}{*}{ Model Soil } & \multicolumn{13}{|c|}{ SDS concentrations in the mobile phase $/ 10^{-3} \mathrm{~mol} \mathrm{dm}^{-3}$} \\
\hline & 8.25 & 8.50 & 8.70 & 8.75 & 8.80 & 9.00 & 9.10 & 9.25 & 9.30 & 9.40 & 9.50 & 9.60 & 9.75 \\
\hline & \multicolumn{13}{|c|}{ capacity factors $\left(k^{\prime}=\left(V_{\mathrm{e}}-V_{\mathrm{m}}\right) / V_{\mathrm{m}}\right)$} \\
\hline Squalene & & 486.08 & & 438.36 & & 301.45 & & 222.91 & & & 186.05 & & \\
\hline Cholesterol & 368.07 & 254.78 & & 184.99 & & 133.79 & & & & & & & \\
\hline Oleic Acid & & 363.41 & 328.11 & & 346.46 & 347.12 & 294.37 & & 240.84 & 217.37 & & & \\
\hline Triolein & & & & 1002.64 & & 773.35 & & 453.4 & & 400.65 & 374.65 & 371.73 & 375.36 \\
\hline $\begin{array}{l}\text { Solvent } \\
\quad \text { Yellow } 5\end{array}$ & & 36.78 & & 27.85 & & 20.64 & & 17.06 & & & & & \\
\hline
\end{tabular}

coefficients: the partition coefficient of the solute between micelles and water $\left(P_{\mathrm{mw}}\right)$, between the stationary phase and water $\left(P_{\mathrm{sw}}\right)$ and between micelles and the stationary phase $\left(P_{\mathrm{ms}}\right)$. The partition coefficients $P_{\mathrm{sw}}$ and $P_{\mathrm{mw}}$ can be calculated with the following equation proposed by Armstrong et al. (6):

$$
\frac{V_{\mathrm{s}}}{V_{\mathrm{e}}-V_{\mathrm{m}}}=\frac{\nu\left(P_{\mathrm{mw}}-1\right)}{P_{\mathrm{sw}}} C_{\mathrm{m}}+\frac{1}{P_{\mathrm{sw}}}
$$

where $V_{\mathrm{s}}$ is the volume of the stationary phase (the total column volume minus $V_{\mathrm{m}}$ ), $V_{\mathrm{e}}$ is the elution volume, $V_{\mathrm{m}}$ is the volume of the mobile phase (the void volume), $v$ is the partial specific volume of the surfactant in the micelle, and $C_{\mathrm{m}}$ is the concentration of surfactant present in the micelles in $\mathrm{g} / \mathrm{mL}$ (the total concentration of surfactant minus the cmc). To derive Eq. (1), it is assumed that the cmc and the aggregation number or geometry of the micelle are not affected by the small amounts of solute being chromatographed or by increasing the concentration of micelles at lower surfac- tant concentrations (6). The ratio $V_{\mathrm{s}} /\left(V_{\mathrm{e}}-V_{\mathrm{m}}\right)$ calculated for SY5 and each fatty soil is plotted against $C_{\mathrm{m}}$ in Figs. 6 and 7, respectively. Straight lines with a positive slope were obtained in agreement with Eq. (1). Table 3 shows the values of $P_{\mathrm{sw}}$ and $P_{\mathrm{mw}}$ calculated from the intercepts and slopes in Figs. 6 and 7 and the $P_{\mathrm{ms}}$ value obtained from the ratio of $P_{\mathrm{sw}}$ and $P_{\mathrm{mw}}$ for each fatty soil and SY5. We used $v=0.862 \mathrm{~mL} / \mathrm{g}$ for SDS (7) to calculate $P_{\mathrm{mw}}$.

The standard free energy change for the transfer of fatty soil from the substrate (s) into micelles (m) ($\Delta G_{\mathrm{ms}}^{\circ}$ ) can be calculated by the following relation at constant temperature and pressure:

$$
-\Delta G_{\mathrm{ms}}^{\circ}=R T \ln P_{\mathrm{ms}}
$$

where $T$ is the absolute temperature and $R$ is the gas constant. The standard free energy change for the transfer of the fatty soil from water (w) to micelles $\left(-\Delta G_{\mathrm{mw}}^{\circ}\right)$ and from water onto fiber substrate $\left(-\Delta G_{\mathrm{sw}}^{\circ}\right)$ can be 


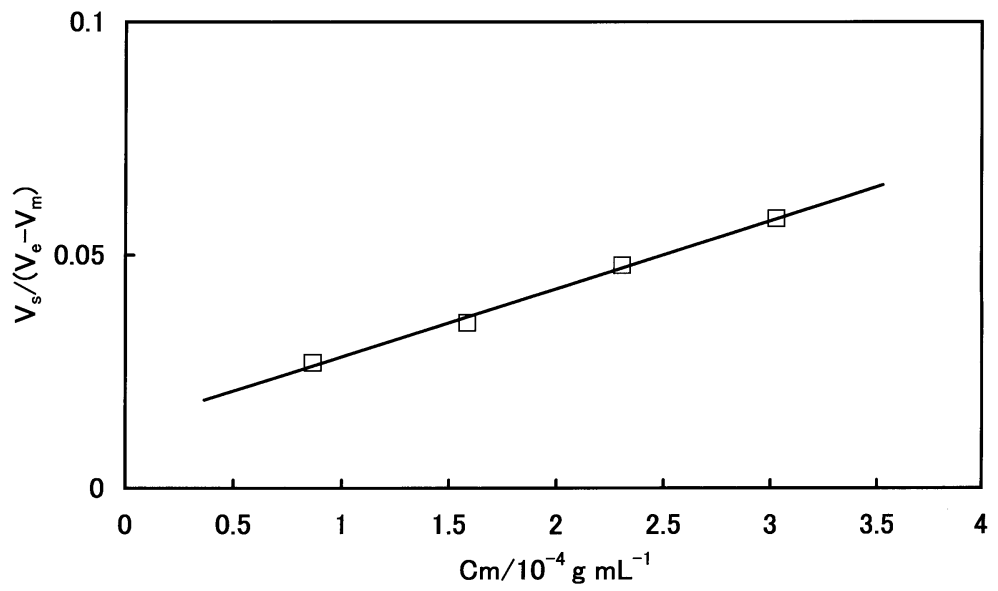

Fig. 6 Plots of the Chromatographic Parameters $V_{\mathrm{s}} /\left(V_{\mathrm{e}}-V_{\mathrm{m}}\right)$ vs. the Concentration of SDS in the Micelle.

Model Soil for Polyester Fabric Column: $\square$; Solvent Yellow 5.

calculated in the same way. The values of $-\Delta G_{\mathrm{ms}}^{\circ}$, $-\Delta G_{\mathrm{mw}}^{\circ}$ and $-\Delta G_{\mathrm{sw}}^{\circ}$ for all fatty soils and SY5 are given in Table 4.

\subsection{Free Energy Change of Fatty Soil Transfer from Water onto Fabric Sub- strate}

In the soil removal process, the $-\Delta G_{\mathrm{sw}}^{\circ}$ value can be considered the standard free energy change of the redeposition of a soil onto the PET substrate after its removal from the substrate into water (non-micellar solution). The $-\Delta G_{\mathrm{sw}}^{\circ}$ values shown in Table 4 are considerably greater for the four fatty soils than for oil-

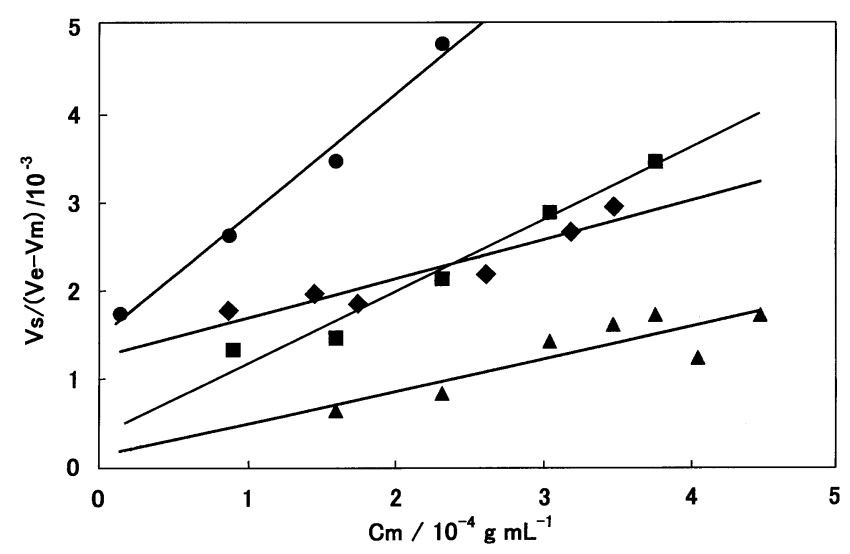

Fig. 7 Plots of the Chromatographic Parameters $V_{\mathrm{s}} /\left(V_{\mathrm{e}}-V_{\mathrm{m}}\right)$ vs. the Concentration of SDS in the Micelle. Model Soil for Polyester Fabric Column: Cholesterol, $\mathbf{\square}$; Squalene, $\diamond$; Oleic acid, $\mathbf{\Delta}$; Triolein. soluble dyestuff SY5. Structurally, the large difference between the four fatty soil molecules and SY5 is the presence of methylene chains in the four fatty soil molecules. It can be inferred that their hydrophobic methylene chains have a high affinity for PET hydrophobic ethylene units, which produces significantly greater re-deposition of the fatty soils than that of SY5 on PET substrate.

The $-\Delta G_{\mathrm{sw}}^{\circ}$ value for triolein, the most hydrophobic of the four fatty soils, is similar in magnitude to the affinity of C. I. Disperse Red 1 for polyester fiber in an aqueous dyeing system $\left(22.59 \mathrm{~kJ} \mathrm{~mol}^{-1}\right)(8)$. These $\Delta G_{\text {sw }}^{\circ}$ values indicate that the re-deposition tendency of the fatty soils onto PET is not negligible. The $-\Delta G_{\mathrm{sw}}^{\circ}$ value for re-deposition of the fatty soils onto PET

Table 3 The Partition Coefficients of Fatty Soils and Oilsoluble Dyestuff between the Micelle and the Polyester Fabric Substrate $\left(P_{\mathrm{ms}}\right)$, between the Micelle and Water $\left(P_{\mathrm{mw}}\right)$, and between the Polyester Fabric Substrate and Water $\left(P_{\mathrm{sw}}\right)$ at $20^{\circ} \mathrm{C}$.

\begin{tabular}{clrcr}
\hline Soil & & $P_{\mathrm{ms}}$ & \multicolumn{1}{c}{$P_{\mathrm{mw}}$} & \multicolumn{1}{c}{$P_{\mathrm{sw}}$} \\
\hline \multirow{4}{*}{ Fatty soil } & Squalene & 9.3 & $2.3 \times 10^{4}$ & 2500 \\
& Cholesterol & 16.4 & $1.1 \times 10^{4}$ & 667 \\
& Oleic Acid & 5.2 & $0.4 \times 10^{4}$ & 769 \\
& Triolein & 4.6 & $4.6 \times 10^{4}$ & 10000 \\
\hline $\begin{array}{c}\text { Oil-soluble } \\
\text { dyestuff }\end{array}$ & Solvent Yellow 5 & 169 & $1.2 \times 10^{4}$ & 74 \\
\hline
\end{tabular}


Table 4 The Standard Free Energy Change $\left(-\Delta G^{\circ}\right)$ for Removal of Fatty Soils from a Fabric Substrate in an Aqueous Micellar Solution of SDS (pH 5.6) at $20^{\circ} \mathrm{C}$.

\begin{tabular}{llccc}
\hline $\begin{array}{c}\text { Substrate } \\
\text { (Fabric) }\end{array}$ & Model Soil & $\begin{array}{c}-\Delta G_{\mathrm{ms}}^{\circ} \\
\left(\mathrm{kJ} \mathrm{mol}^{-1}\right)\end{array}$ & $\begin{array}{c}-\Delta G_{\mathrm{mw}}^{\circ} \\
\left(\mathrm{kJ} \mathrm{mol}^{-1}\right)\end{array}$ & $\begin{array}{c}-\Delta G_{\mathrm{sw}}^{\circ} \\
\left(\mathrm{kJ} \mathrm{mol}^{-1}\right)\end{array}$ \\
\hline \multirow{4}{*}{ Polyester } & Cholesterol & 6.81 & 22.65 & 15.83 \\
& Squalene & 5.42 & 24.44 & 19.04 \\
& Oleic Acid & 4.01 & 20.19 & 16.18 \\
& Triolein & 3.71 & 26.14 & 22.42 \\
\cline { 2 - 5 } & Solvent Yellow 5 & 12.50 & 22.99 & 10.49 \\
\hline
\end{tabular}

increases with increases in the length and number of their hydrophobic chains as follows: cholesterol $<$ oleic acid $<$ squalene $<$ triolein. This suggests that the strong hydrophobic interaction between methylene groups of the fatty soil molecules and ethylene units in PET causes an increase in re-deposition of these fatty soils from water onto PET. Thus, the $-\Delta G_{\mathrm{sw}}^{\circ}$ values shown in Table 4 indicate that complete removal of these types of hydrophobic soils from nonpolar substrates like PET with aqueous non-micellar solution is impossible.

We have reported that with a filament yarn substrate, the $-\Delta G_{\mathrm{sw}}^{\circ}$ value of SY5 is $12.64 \mathrm{~kJ} \mathrm{~mol}^{-1}$ for polyester (3). The $-\Delta G_{\mathrm{sw}}^{\circ}$ value for the PET filament yarn substrate is greater than those for the fabric substrate used in the present study. The difference in $-\Delta G_{\mathrm{sw}}^{\circ}$ values between the fabric and filament substrates suggests that the physico-chemical properties of the surfaces differ between the polyester filament yarn and fabric substrates. The $\zeta$-potential for the filament yarn and fabric substrates was determined in $1 \times 10^{-3} \mathrm{~mol} \mathrm{dm}^{-3} \mathrm{NaCl}$ aqueous solution to estimate the electrical interfacial properties of these surfaces. The values of $-\zeta$-potentials for the PET fabric and filament yarn were $23.13 \mathrm{mV}$ and $26.82 \mathrm{mV}$, respectively. The value of $-\zeta$-potentials for the PET polyester fabric was slightly less than that of the PET filament yarn. These results suggest that electrostatic attraction between the positively charged SY5 and the negatively charged polyester substrate is greater for the filament yarn than for the fabric, and this increases the re-deposition of SY5 onto filament yarn substrate. We conclude that the $-\Delta G_{\mathrm{sw}}^{\circ}$ value depends strongly on the physico-chemical properties of the surface used as substrate.

\subsection{Free Energy Change of Fatty Soil Transfer from Water into Micelles}

For solubilization of anthracene and Sudan IV into micellar solutions like those used in the present study, S. Kumar et al. (9) reported values of $22.33 \mathrm{~kJ} \mathrm{~mol}^{-1}$ and $25.83 \mathrm{~kJ} \mathrm{~mol}^{-1}\left(30^{\circ} \mathrm{C}\right)$, respectively, as measured spectrophotometrically. These values are similar in magnitude to the $-\Delta G_{\text {mw }}^{\circ}$ for cholesterol, SY5 and triolein. Moreover, the $-\Delta G_{\mathrm{mw}}^{\circ}$ value for triolein is the same order of magnitude as that for micelle-water partition coefficients of $n$-hexane $\left(27.65 \mathrm{~kJ} \mathrm{~mol}^{-1}, 25^{\circ} \mathrm{C}\right)(10)$ and the solubilization of hemicyanine dye, which has 5 carbons in the alkyl groups of dialkyl amino stilbazolium butyl sulfonate $\left(25.53 \mathrm{~kJ} \mathrm{~mol}^{-1}, 25^{\circ} \mathrm{C}\right)(11)$, in a micellar solution of the same surfactant used in the present study. Thus, the $-\Delta G_{\mathrm{mw}}^{\circ}$ values in Table 4 are reasonable.

The $-\Delta G_{\text {mw }}^{\circ}$ values for the fatty soils and SY5 are much grater than the $-\Delta G_{\mathrm{sw}}^{\circ}$ values, indicating that the fatty soils and SY5 have a much grater affinity for SDS micelles than for the PET substrate. As the number of carbon atoms of the fatty soil molecules increases, the $-\Delta G_{\mathrm{mw}}^{\circ}$ values increase in the following order: oleic acid $<$ cholesterol $<$ squalene $<$ triolein. The larger $-\Delta G_{\mathrm{mw}}^{\circ}$ values for the increased number of fatty soil carbon atoms suggest increased hydrophobic interaction with the SDS micelles. Moreover, our results are consistent with those of earlier studies showing that the negative standard free energy change for solubilization of a solubilizate molecule in several aqueous surfactant micellar systems increases with increases in the number of solubilizate carbon atoms (11-13). These thermodynamic parameters suggest that triolein, which is the most hydrophobic and has the greatest $-\Delta G_{\mathrm{mw}}^{\circ}$ value 
of the four fatty soils, is buried deep within the core of the micelle because of the strong hydrophobic interaction between the fatty soil and SDS. In contrast, these same parameters suggest that oleic acid, which has a carboxyl group that can become hydrated, is located at an outer region of the micelle where water molecules tend to penetrate.

The SY5 molecule has two less carbon atoms than oleic acid. The value of $-\Delta G_{\mathrm{mw}}^{\circ}$ for SY5, however, is greater than that for oleic acid. It can be inferred that strongly attractive interactions between the positively charged SY5 and the negatively charged micelles produced a lager $-\Delta G_{\mathrm{mw}}^{\circ}$ value for SY5 than for electronegative oleic acid and much the same $-\Delta G_{\text {mw }}^{\circ}$ value for cholesterol which has a hydroxyl group and 11 more carbon atoms than SY5 has. The partitioning of the fatty soils and SY5 between aqueous bulk phase and the micellar phase depends on their hydrophobicity as well as their polarity.

\section{4-4 Free Energy Change of Fatty Soil Transfer from Fabric Substrate into Micelles}

Oily soil removal by surfactant micellar solution is a two-stage process: transfer of the soil from substrate into water (non-micelle solution) and then from water into micelles (3). After its removal from substrate into water, the soil will transfer either into micelles or back onto the substrate. For all four fatty soils and SY5, the $-\Delta G_{\text {mw }}^{\circ}$ values are greater than the $-\Delta G_{\text {sw }}^{\circ}$ values (Table 4); thus, the tendency of the fatty soils and SY5 to migrate from water into micelles (soil removal) is greater than that from water onto PET substrate (redeposition). Therefore, the total soil removal process seems to proceed.

The $-\Delta G_{\mathrm{ms}}^{\circ}$ values of the four fatty soils are much smaller than that of the oil-soluble dyestuff, SY5 (Table 4) because the $-\Delta G_{\text {sw }}^{\circ}$ value for the SY5 is much smaller than those for the fatty soils and because the difference between the $-\Delta G_{\mathrm{mw}}^{\circ}$ values of the SY5 and those of the fatty soils are not very large. Therefore, we conclude that fatty soil removal from PET is difficult because of the greater tendency of the fatty soil to redeposit onto PET. Conversely, SY5 is easily removed from PET because the tendency of SY5 to redeposit onto PET is much lower than that of the four fatty soils.

The $-\Delta G_{\mathrm{ms}}^{\circ}$ value for triolein is half that of cholesterol and is the smallest of the four fatty soils (Table 4).
In contrast, the $-\Delta G_{\mathrm{sw}}^{\circ}$ value for triolein is 1.4 times greater than that for cholesterol and is the greatest of the four fatty soils. Triolein is the most difficult constituent to remove because it has the greatest tendency to redeposit onto PET. In comparison with triolein, removal of squalene from PET is easier, and removal of cholesterol from PET is easiest because the tendency of these soils to redeposit onto PET is low. With the exception of oleic acid, these results are in agreement with the ease of removal of fatty soil at $50^{\circ} \mathrm{C}$ (14) or skin sebum constituents at $35^{\circ} \mathrm{C}$ (1) from PET fabric by an alkaline detergent solution.

Because oleic acid, which has a carboxyl group, is the most polar of the four fatty soils, ease of removal from PET is expected. However, because oleic acid has the least tendency to solubilize and the tendency to redeposit onto PET is greater than that of cholesterol, removal of oleic acid is more difficult than removal of squalene or cholesterol. The work of adhesion (Wa) of fatty acids and triglycerides with alkyl groups of 12 to 18 carbons onto PET film in water has been calculated by applying the values of surface tension components for those fatty soils, polyester substrate and water (15). The Wa value for both fatty acids and triglycerides with 12 or 16 carbons at $20^{\circ} \mathrm{C}$ are almost the same, whereas the Wa value is slightly larger for triglycerides than for fatty acids, when the number of carbon atoms is 14 or 18. These facts are consistent with our thermodynamic results showing the tendency toward easy removal of oleic acid and triolein.

\section{Conclusions}

The removal of fatty soil from fabric by aqueous surfactant solution depends on the free energy change of the transfer of the soil from a fabric substrate into micelles $\left(-\Delta G_{\mathrm{ms}}^{\circ}\right)$. Our findings indicate that the four fatty soils have high affinity for PET; thus, the high tendency of the fatty soils to redeposit onto PET makes removal difficult. Of the four fatty soils, triolein has the smallest $-\Delta G_{\mathrm{ms}}^{\circ}$ value and the largest value of free energy change of the soil transfer from water (nonmicellar solution) onto fabric substrate $\left(-\Delta G_{\text {sw }}^{\circ}\right)$. Therefore, triolein is the most difficult of the four fatty soils to remove from PET because it has the greatest tendency to redeposit. Cholesterol is the easiest soil to remove from PET because it has the least tendency to redeposit. 


\section{Acknowledgment}

This work was supported in part by Grants-in-Aid for Scientific Research (C-2) from the Ministry of Education, Culture, Sports, Science and Technology of Japan.

\section{References}

1. J. MINO, Removal of Oily Soil in Detergent Processes, in Detergency (W.G. CUTLER and E. KISSA, eds.), Marcel Dekker, New York, pp. 513-515 (1987).

2. E. KISSA, Fatty Soils from Skin, in Detergency (W.G. CUTLER and E. KISSA, eds.), Marcel Dekker, New York, pp. 8-11 (1987).

3. H. TAGAYA, Y. YOSHIDA and K. HIGASHITSUJI, A New Approach to the Study of the Mechanism of Oily Soil Removal by Means of Liquid Chromatography with a Filament Yarn Column, J. Oleo Sci., Vol. 51, 789-798 (2002).

4. H. TAGAYA, Y. YOSHIDA and K. HIGASHITSUJI, The Elucidation of the Mechanism for Oily Soil Removal Using Liquid Chromatography with a Fiber Powders Column, J. Chem. Soc. Jpn, (12), 811-816 (1998).

5. H. TAGAYA, K. HIGASHITSUJI, Y. TANIZAWA and N.TSUTIKAWA, Paper Chromatographic Observation on the Effects of Surface Active Agents to Remove the Soil on Fiber Assemblies, J. Jpn Oil Chem. Soc., Vol. 41, 317-324 (1992).

6. D.W. ARMSTRONG and F. NOME, Partitioning Behavior of Solutes Eluted with Micellar Mobile Phases in Liquid Chromatography, J. Anal. Chem., Vol. 53, 1662-1666 (1981).

7. P. MUKERJEE, The Partial Specific Volume and Density of Micelles of Association Colloidal Electrolytes, J. Phys. Chem.,
Vol. 66, 1733-1735 (1962).

8. N. KUROKI, Theoretical Chemistry of Dyeing, Maki-Shyoten, Tokyo, p. 497 (1961).

9. S. KUMAR and H.N. SINGH, Competitive Solubilization of Sudan IV and Anthracene in Micellar Systems, Colloids Surfaces, Vol. 69, 1-4 (1992).

10. R. NAGARAJAN, M.A. CHAIKO and E. RUCKENSTEIN, Locus of Solubilization of Benzene in Surfactant Micelles, $J$. Phys. Chem., Vol. 88, 2916-2922 (1984).

11. S.S. SHAH, G.M. LAGHGARI, K. NAEEM and S.W.H. SHAH, Partition Coefficient of Amphiphilic Hemicyanine Dyes between the Aqueous and the Micellar Phase of Sodium Dodecyl Sulfate by Differential Absorbance Spectroscopy, Colloids Surfaces A, Vol. 143, 111-115 (1998).

12. Y. MOROI, K. MITUNOBU, T. MORISUE, Y. KADOBAYASI and M. SAKAI, Solubilization of Benzene, Naphthalene, Anthracene, Pyrene in 1-Dodecanesulfonic Acid Micelle, J. Phys. Chem., Vol. 99, 2372-2376 (1995).

13. G. MARANGONI and J.C.T. KWAK, Comparison of Experimental Methods for the Determination of the Partition Coefficients of n-Alcohols in SDS and DTAB Micelles, in SOLUBILIZATION IN SURFACTANT AGGREGATES (D.S. CHRISTIAN and J.F. SCAMEHORN, eds.), Marcel Dekker, New York, pp. 455-490 (1995).

14. S. YOKOYAMA, S. SHIMAUCHI and H. MIZUSHIMA, Studies on Fatty Soil on Polyester Fiber Using a Radioctive Tracer. (I) Comparison of the Washing Removability of Various Fatty Soils on Polyester and Celluloisic Fabrics, Sen-I Gakkaishi, Vol. 23, 444-448 (1967).

15. M. SAITO, M. OTANI and A. YABE, Work of Adhesion of Oil Dirt and Correlation with Washability, Textile Res. J., Vol. 55, 157-164 (1985). 